\title{
High-accuracy surface measurement through modelling of the surface transfer function in interference microscopy
}

Rong Su, Matthew Thomas, Mingyu Liu, Jeremy Coupland, Richard Leach

Rong Su, Matthew Thomas, Mingyu Liu, Jeremy Coupland, Richard Leach, "High-accuracy surface measurement through modelling of the surface transfer function in interference microscopy," Proc. SPIE 11102, Applied Optical Metrology III, 1110205 (3 September 2019); doi: 10.1117/12.2528911

EDent: SPIE Optical Engineering + Applications, 2019, San Diego, California, United States 


\title{
High-accuracy surface measurement through modelling of the surface transfer function in interference microscopy
}

\author{
Rong Su${ }^{1 *}$, Matthew Thomas ${ }^{1}$, Mingyu Liu ${ }^{1}$, Jeremy Coupland ${ }^{2}$, Richard Leach ${ }^{1}$ \\ ${ }^{1}$ University of Nottingham, Nottingham NG8 1BB, UK \\ ${ }^{2}$ Loughborough University, LE11 3TU, UK
}

\begin{abstract}
Surfaces featuring complex topographies, such as high slope angles, large curvatures and high aspect-ratio structures on both macro- and micro-scales, present significant challenges to optical measuring instruments. Here we demonstrate a method to characterise and correct the three-dimensional surface transfer function (3D STF) of a coherence scanning interferometer (CSI). Slope-dependent errors present in the original measurements are reduced after phase inversion of the 3D STF, and the final results agree with traceable contact stylus measurements within the $30 \mathrm{~nm}$ reproducibility of the stylus measurements. This method enables in-situ compensation for errors related to aberrations, defocus and diffraction.
\end{abstract}

Keywords: Coherence scanning interferometry, error correction, transfer function, metrology, surface topography

\section{INTRODUCTION}

Surface topography has a profound effect on the function of a manufactured part, such as medical implants, micro-needles and fluidic channels, gears, turbine blades and freeform optics [1]. Also, surface topography is highly sensitive to changes in a manufacturing process. Therefore, measurement of the surface can be used to control product quality and monitor a process of manufacture by providing feedback (Figure 1). However, due to various functional specifications and the characteristics of manufacturing processes, surface topographies can be diverse and complex. For example, additive manufactured surfaces have deterministic and random features caused by the thermal distortions of a highly energetic process and by the irregularity of partially melted particles that adhere to the part surface [2,3], and manufacturing of aspheric and freeform surfaces often leaves tooling marks that are primarily in the mid-spatial-frequency region $[4,5]$. Such surfaces often feature high slope angles, large curvatures and high aspect-ratio structures on both macro- and microscales.

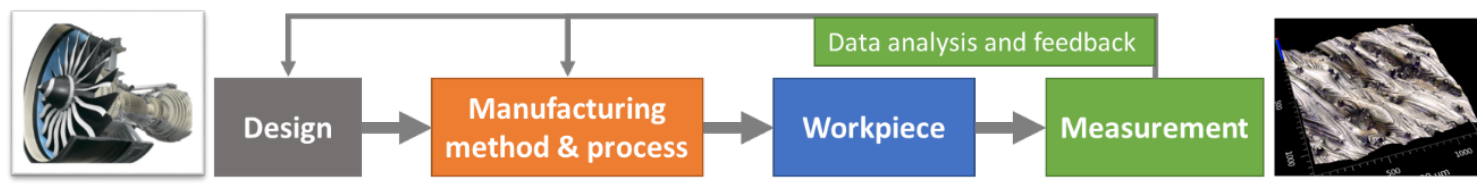

Figure 1. Schematic illustration of a typical closed-loop process for manufacturing and measurement.

Optical measuring systems [6] outperform traditional mechanical methods in that they reduce surface damage, have higher resolutions (except for the case of atomic force microscope), can allow improved accessibility, and operate at high speed, therefore, allowing dense 3D point cloud capture in production processes. However, the complex surfaces significantly challenge the use of optical measuring techniques [7]. The current calibration framework and methods for optical surface measuring instruments need to be improved to respond to the increasing geometrical complexity of functional surfaces.

Interference microscopy is one of the most accurate techniques to offer non-contact, high-speed and high-resolution measurement of three-dimensional (3D) surface topography [6-9]. The major modalities of interference microscopy include coherence scanning interferometer (CSI) and phase-shifting interferometry (PSI) [6,8]. In this paper we will focus on the CSI in which the interference phenomena can take place only within a window of a few micrometres of the zero optical path difference of the interferometer as the instrument scans along the optical axis of the system.

A recent study shows that an ideal CSI working at the diffraction-limited condition should be capable of achieving sub-nanometre accuracy when measuring surfaces with varying slopes [10]. However, if the system has not been well calibrated and characterised, lateral distortion, optical aberrations and defocus can significantly degrade the resolving

Applied Optical Metrology III, edited by Erik Novak, James D. Trolinger, Proc. of SPIE Vol. 11102, $1110205 \cdot$ (c) 2019 SPIE · CCC code: 0277-786X/19/\$21 · doi: 10.1117/12.2528911 
power of the microscope and the measurement accuracy when measuring a surface with complex geometries. For such surfaces, measurement errors can be much larger than the noise level of the instrument [11-13].

Current calibration methods [14] do not identify slope-dependent errors in CSI as the understanding of the optical instrument response to surfaces with complex geometries is insufficient [7]. A promising method to quantify the instrument response of a CSI is through the evaluation of its three-dimensional surface transfer function (3D STF, referred to in [10,15 -17] as the 3D transfer function). The 3D optical imaging of an object can be considered as a 3D shift-invariant filtering process applied to the source distribution $[15,18]$. Within the framework of scalar diffraction theory, the source distribution at the surface is determined by the spatial distributions of the refractive indices of the object and its surrounding medium (usually air), and the complex amplitudes of the incident and scattered electromagnetic fields. However, the source distribution is usually unknown because it is difficult to accurately find the scattered field without using computationally intensive rigorous solutions to surface scattering problems.

Alternatively, it is possible to linearise the surface-to-image process by using an approximate approach for solving the scattering problem, such as the Kirchhoff theory of scattering $[19,20]$, where the surface is divided into locally-flat elements. Based on this approximation, the "foil model" was derived [15] which describes the optical imaging as a 3D linear filtering operation applied to the surface rather than the source distribution, assuming that the object is a homogeneous medium. The associated transfer function is called the 3D STF, as it describes the property of transferring the surface topography to the optical field that is recorded by the instrument, whereas the more familiar optical transfer function (OTF) describes the transfer property from field to field. The Fourier transform of the 3D STF gives the instrument's impulse response - the 3D point spread function (3D PSF).

In our previous papers, a method for experimentally determining the 3D STF of a CSI, by measuring a precision microsphere which has a diameter much larger than a wavelength, has been proposed and evaluated [16,21]. The integration of the 3D STF over the axial spatial frequency for each lateral spatial frequency results in the in-pupil STF [21,22], which is similar to the concept of in-pupil (2D) OTF that can be found at the back focal plane of the objective lens [23]. The effects of the form error of the precision microsphere on the 3D STF have been reported [10], as well as the effects of defocus in CSI [17].

In this work, we demonstrate that by correcting the 3D STF of a CSI through phase-inversion filtering, the form and $2 \pi$ errors can be effectively reduced. To validate the method, optical measurements are compared with results obtained using a stylus instrument.

\section{METHODS}

The detailed procedure for characterisation of the 3D STF of CSI can be found elsewhere $[10,16,21]$. Here, the procedure is briefly illustrated in Figure 2. We first acquire the 3D CSI fringe data of the precision sphere and store the image stack in the usual way. Given that the sphere diameter is known, the foil function/model of the spherical cap can be generated according Eq. 35 in [15]. Taking the Fourier transform of the fringe data and the foil function gives the spectra of the spatial frequency components. Then the 3D STF for the CSI at the time of characterisation can be calculated by dividing the spectra of the fringe by the foil function.

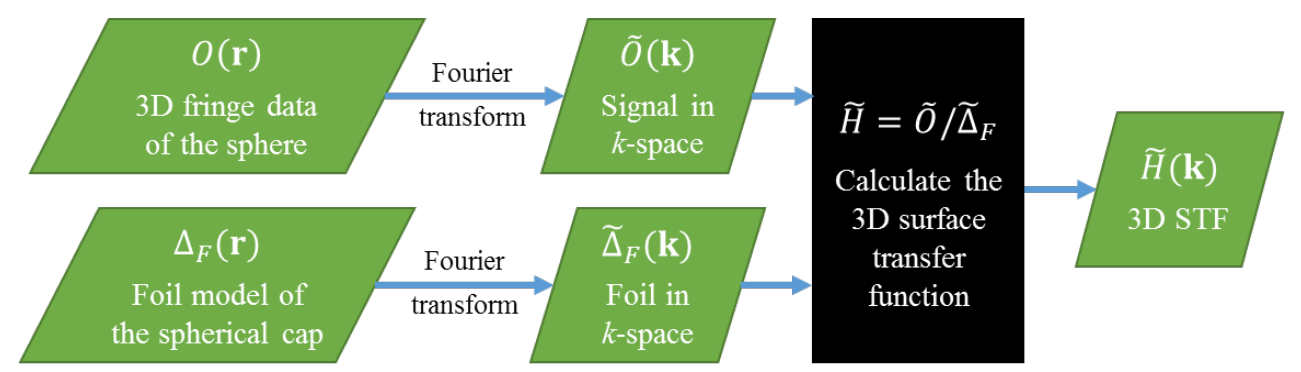

Figure 2. Schematic characterisation procedure of 3D STF of a CSI. $\mathbf{k}$ represents the spatial frequency.

The instrument used in this work is a CSI with a Mirau objective lens ( 0.55 NA), $0.174 \mu \mathrm{m}$ lateral sampling distance, $1000 \times 1000$ lateral sampling points and central wavelength $0.56 \mu \mathrm{m}$. The silica microspheres for the characterisation were produced by melting non-spherical $\mathrm{SiO}_{2}$ particles in plasma to form spherical droplets, and then cooled to obtain solid spheres [21]. The diameter of the sphere was measured as the distance between the top of the sphere and the surface of the optical flat. The influence of the accuracy of the diameter and sphericity on the 3D STF has been demonstrated elsewhere 
[10]. The spheres' diameters are $39.4 \mu \mathrm{m}$ and $45.0 \mu \mathrm{m}$, respectively, and the standard deviations of the ten repeated diameter measurements are less than $10 \mathrm{~nm}$.

To compensate errors in the CSI measurement, the fringe data of a surface can be modified by multiplying with an inverse filter in the spatial frequency domain, followed by an inverse Fourier transform [21]. The inverse filter is calculated

through the phase inversion of the measured $3 \mathrm{D} \operatorname{STF}$ as $\hat{H}_{i n v}(\mathbf{k})=\exp \left[-j \cdot \angle H_{F}^{\circ}(\mathbf{k})\right]$. Using the frequency-domain analysis method [24], surface topographies calculated from the original and filtered CSI signals are compared.

The effectiveness of the inverse filtering is tested by using a sinusoidal surface (Rubert reference specimen 525) with a nominal pitch of $135 \mu \mathrm{m}$ and nominal peak-to-valley amplitude of $19 \mu \mathrm{m}$, and a different sphere that is produced using laser-morphing technique [25,26]. The radius of the sphere is $102.0 \mu \mathrm{m}$, determined using a well-established interferometric radius measurement procedure [21]. A stylus instrument ( $2 \mu \mathrm{m}$ tip radius) was used to measure the sinusoidal surface for comparison.

\section{RESULTS}

Figure 3 shows the experimentally determined 3D STF of the CSI. To minimise the characterisation error that can be caused by the size error, asphericity of the sphere or other statistical error sources, we measured the two silica precision microspheres at four rotation angles with three repeats. The mean value of the subsequent twenty-four measured 3D STFs were then obtained. The mean value of the standard deviations (across the pupil) of the magnitudes and phases of the corresponding in-pupil STFs are 0.004 and $0.06 \mathrm{rad}$, respectively, as plotted in Figure 3(K) and (L). The small variations in both magnitudes and phases provide evidence that the measured STF is stable and independent of the spheres, and the sphere form errors are sufficiently small.

The 3D STF is a complex-valued quantity. Its magnitude determines the passband of spatial frequencies of the CSI, and the peak modulation of the magnitude is located at approximately the spatial frequency $2 / \lambda_{0}$ on the $k_{z}$ axis, where $\lambda_{0}$ is the central wavelength of the light source. The 3D STF of an ideal system should in principle be rotationally symmetric about the $k_{z}$ axis.

As shown in Figure 3, the magnitude of the experimental 3D STF slightly deviates from the ideal one. The degraded magnitude is likely to be the effect of a combination of spherical aberration, defocus and the central obstruction due to the presence of the reference mirror in the optical axis of the Mirau objective.

The phase plots of the 3D STF are shown next to their corresponding magnitudes. The phase of the real instrument departs from zero, i.e. the ideal (diffraction-limited) case, due to the presence of optical aberrations. The departure and variation of the phase become relatively larger at the edges of the passband but its impact on the measurement result is limited as the corresponding magnitude is small.

The asymmetry and distortion in both magnitude and phase is probably caused by the tilt and decentration of the optical components and other asymmetric aberrations in the optical system, and indicates that the optical system would perform differently along different directions in terms of resolution and measurement accuracy. 

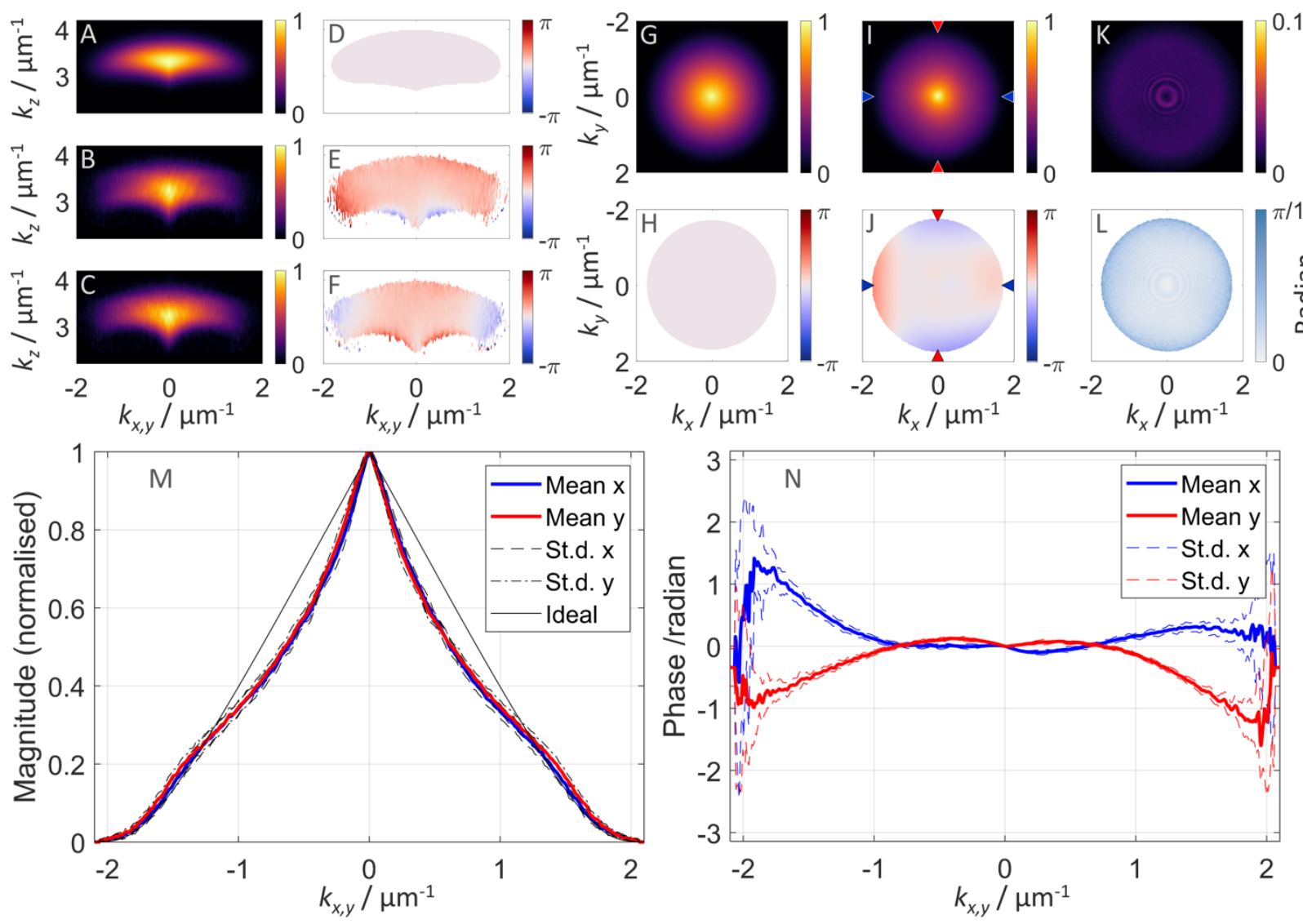

Figure 3. 3D STF of the CSI determined using two precision microspheres. (A) Cross-sectional slice (at either $k_{y}=0$ or $k_{x}=$ 0 ) of the normalised magnitude of the ideal 3D STF. (B, C) Experimental counterparts of (A) at $k_{y}=0$ and $k_{x}=0$, respectively. (D, E and F) Corresponding slices of the phases. (G, H) Magnitude and phase of the ideal in-pupil STF. (I, J) Mean of the magnitudes and phases of the experimental in-pupil STFs obtained from 24 measured 3D STFs. (K, L) Standard deviations of the magnitudes and phases of the experimental in-pupil STFs. (M, N) Profiles of the magnitudes and phases of the experimental in-pupil STFs in the two orthogonal lateral directions, respectively. The profiles are taken along the $k_{x}$ and $k_{y}$ axes as marked in (I, J). The magnitude for the diffraction-limited case is plotted as a black line for reference.

Figure 4 shows the CSI measurement of the laser-morphed sphere before the after the inverse filtering. The real form error of the sphere is revealed after the inverse filtering which compensates the optical aberration in the CSI. It is known that there is an orthogonal anisotropy of the spherical form in the laser-manufactured spheres, i.e. the radius of the sphere slightly varies along two orthogonal horizontal directions. This problem is not surprising as the laser-morphing process started with intrinsically asymmetric conditions, such as thermal gradient, asymmetric material geometry and possible asymmetry in the heating profile. The diameters of the best-fit spheres are $102.1 \mu \mathrm{m}$ and $102.0 \mu \mathrm{m}$, respectively, before and after the error compensation. 

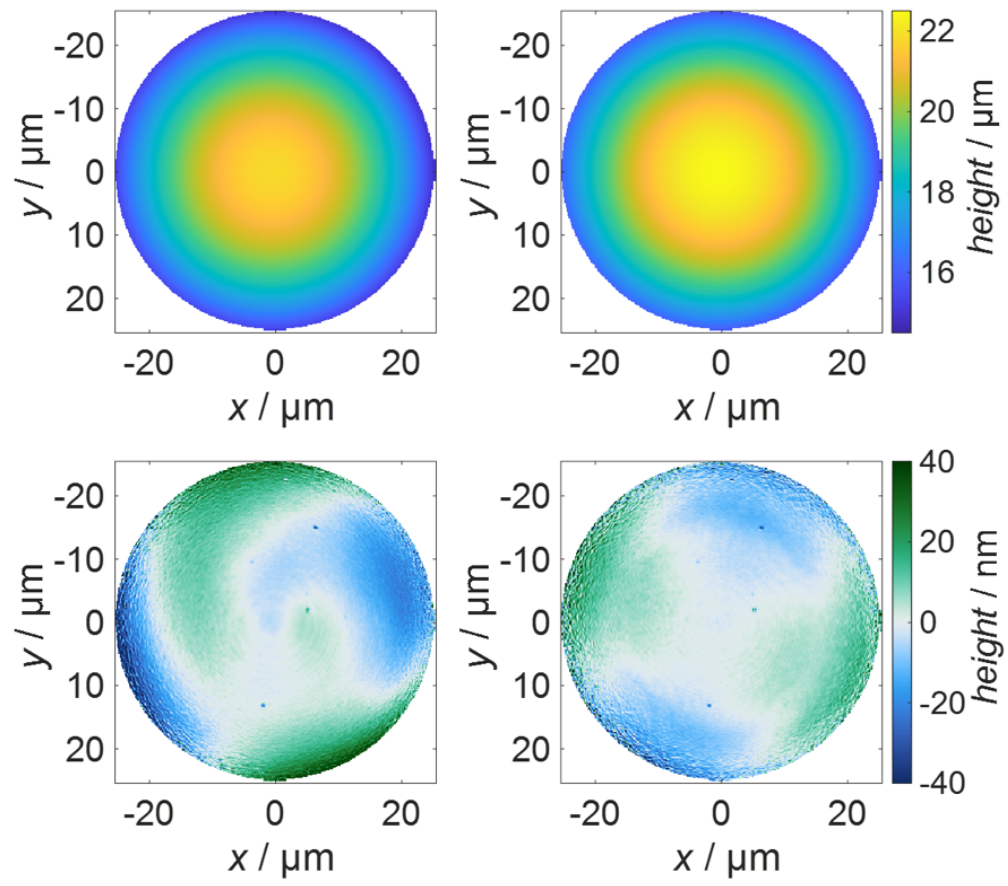

Figure 4. Comparison of the CSI measurements of the laser-morphed sphere before and after inverse filtering. Top row: height maps of the measured spherical cap. Bottom row: height deviations of the measured spherical cap after removing the best-fit sphere. Left column: before inverse filtering; Right column: after inverse filtering.

Figure 5 shows the comparison of the CSI- and stylus-measured profiles of the sinusoidal surface. The CSI result is obtained based on the coherence envelope and phase of the fringes in order to achieve the lowest noise level. However, incorrect estimation of the fringe order may cause $2 \pi$ errors in CSI $[11,27]$. As shown in Figure 5, $2 \pi$ errors appear on the high slope region of the surface, where the maximum slope angle is approximately $31^{\circ}$, corresponding to a lateral spatial frequency of approximately $1.8 \mu \mathrm{m}^{-1}$, which approaches the theoretical limit of the acceptance angle of the lens and the edge of the STF, where the phase distortion is large. Such surfaces may significantly challenge the metrological capability of CSI with an NA of 0.55 .

After the inverse filtering, the $2 \pi$ errors are effectively reduced. The mean value of the standard deviations of the differences between the stylus- and CSI-measured profiles before and after the inverse filtering are $76 \mathrm{~nm}$ and $24 \mathrm{~nm}$, respectively. Note that the reproducibility of the stylus measurement is of the order of $30 \mathrm{~nm}$ [21]. 

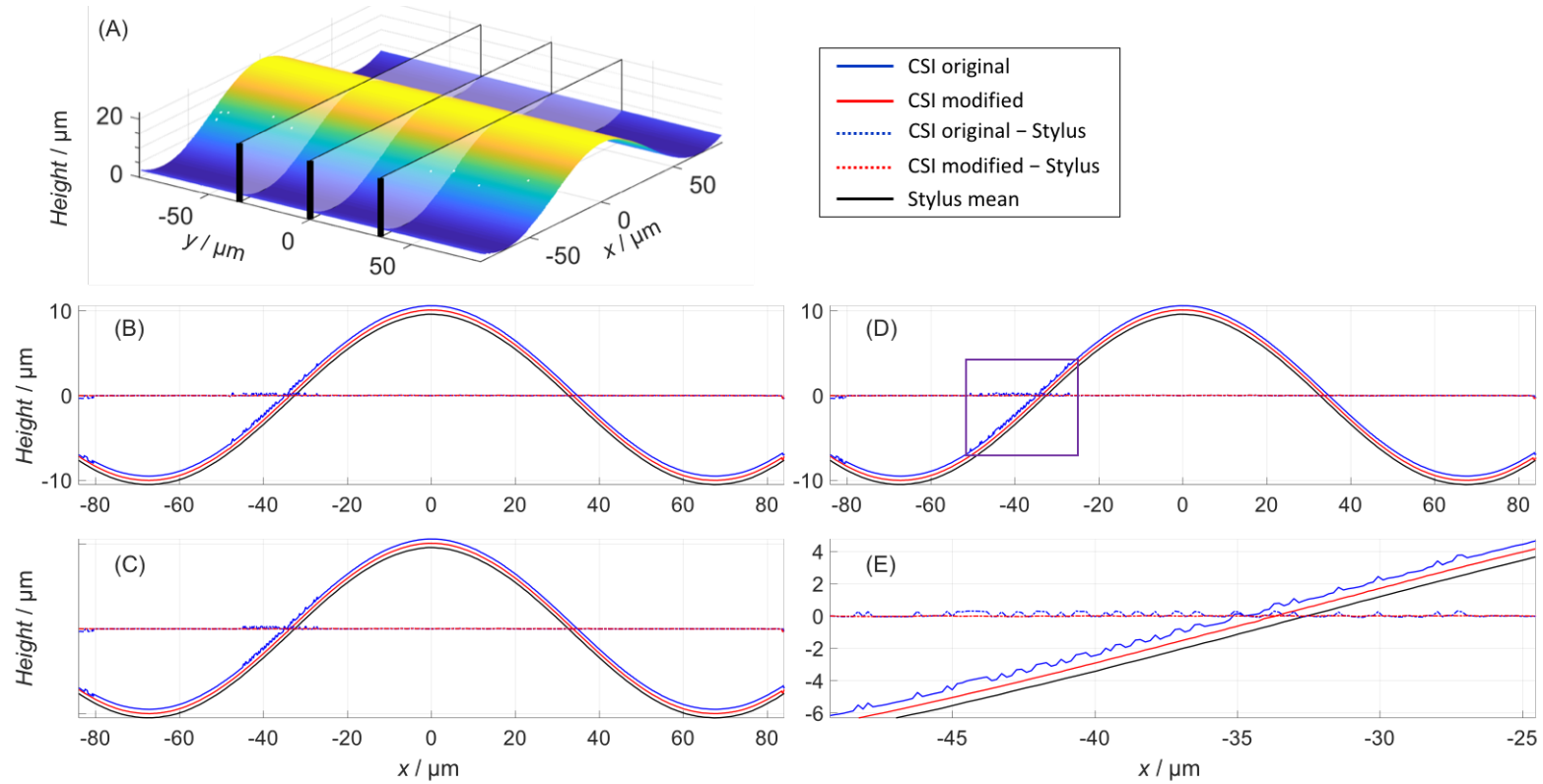

Figure 5. Comparison of the CSI and stylus measurements of the sinusoidal surface (R525). (A) 3D plot of the CSI-measured areal topography. The profiles are extracted at (B) $y=-35 \mu \mathrm{m},(\mathrm{C}) y=0 \mu \mathrm{m}$, (D) $y=35 \mu \mathrm{m}$. (E) Enlarged view of the highlighted region in (D).

\section{CONCLUSION}

In this paper we provide experimental evidence to verify the foil model in CSI for surface measurement by demonstrating the characterisation of the 3D STF using two precision spheres, and show that the inverse filtering based on phase inversion of the 3D STF can improve the CSI measurements of surfaces with varying slopes and spatial frequencies. By using the foil model-based characterisation and error correction approach, the accuracy of CSI that offers sub-nanometre level repeatability can be further enhanced, making this technique suitable when measuring complex functional surfaces produced by advanced manufacturing processes.

\section{ACKNOWLEDGEMENTS}

The authors gratefully acknowledge Dr Peter de Groot for helpful discussions, Prof. Yves Bellouard from EPFL, Switzerland for providing the laser-morphed sphere for testing and Dr Christof Pruss from Institut für Technische Optik, Universität Stuttgart for measuring the diameter of the sphere. Thanks to Dr Peter Kovesi for the use of the colour maps used in Figures 3 and 4 which have been released under a Creative Commons Attribution License (CC BY 4.0). This work was supported by the Engineering and Physical Sciences Research Council (EPSRC) (EP/M008983/1) and the European Union's Horizon 2020 Research and Innovation Programme (MNR4SCell, 734174).

\section{REFERENCES}

[1] Bruzzone, A. A. G., Costa, H. L., Lonardo, P. M. and Lucca, D. A., "Advances in engineered surfaces for functional performance," CIRP Annals 57(2), 750-769 (2008).

[2] Leach, R. K., Bourell, D., Carmignato, S., Donmez, A., Senin, N. and Dewulf, W., "Geometrical metrology for metal additive manufacturing," CIRP Annals, in press (2019).

[3] Gomez, C., Su, R., Thompson, A., DiSciacca, J., Lawes, S. and Leach, R. K., "Optimization of surface measurement for metal additive manufacturing using coherence scanning interferometry," Opt. Eng. 56(11), 111714 (2017).

[4] Tamkin, J. M. and Milster, T. D., "Effects of structured mid-spatial frequency surface errors on image performance," Appl. Opt. 49(33), 6522-6536 (2010). 
[5] Deck, L. L. and Evans, C., "High performance Fizeau and scanning white-light interferometers for mid-spatial frequency optical testing of free-form optics," Proc. SPIE 5921, 59210A (2005).

[6] Leach, R. K., [Optical Measurement of Surface Topography], Springer, Berlin, Heidelberg (2011).

[7] Leach, R. K., de Groot, P. and Haitjema, H., "Infidelity and the calibration of surface topography measuring instruments," Proc. 33rd Annual Meeting of American Society of Precision Engineering, Las Vegas, USA (2018).

[8] de Groot, P., "Principles of interference microscopy for the measurement of surface topography," Adv. Opt. Photon. 7(1), 1-65 (2015).

[9] de Groot, P., de Lega, X. C., Su, R. and Leach, R. K., "Does interferometry work? A critical look at the foundations of interferometric surface topography measurement," Proc. of SPIE Vol. 11102, 11102-15 (2019).

[10] Su, R., Wang, Y., Coupland, J. M. and Leach, R. K., "On tilt and curvature dependent errors and the calibration of coherence scanning interferometry," Opt. Express 25(4), 3297-3310 (2017).

[11] Gao, F., Leach, R. K., Petzing, J. and Coupland, J. M., "Surface measurement errors using commercial scanning white light interferometers," Meas. Sci. Technol. 19(1), 015303 (2007).

[12] Rhee, H.-G., Vorburger, T. V., Lee, J. W. and Fu, J., "Discrepancies between roughness measurements obtained with phase-shifting and white-light interferometry," Appl. Opt. 44(28), 5919-5927 (2005).

[13] Vorburger, T. V., Rhee, H.-G., Renegar, T. B., Song, J.-F. and Zheng, A., "Comparison of optical and stylus methods for measurement of surface texture," Int. J. Adv. Manuf. Technol. 33(1), 110-118 (2007).

[14] Leach R. K., Giusca C. L., Haitjema, H., Evans, C. and Jiang, X., "Calibration and verification of areal surface texture measuring instruments," CIRP Annals 64(2), 797-813 (2015).

[15] Coupland, J. M., Mandal, R., Palodhi, K. and Leach, R. K., "Coherence scanning interferometry: linear theory of surface measurement," Appl. Opt. 52(16), 3662-3670 (2013).

[16] Mandal, R., Coupland, J. M., Leach, R. K. and Mansfield, D., "Coherence scanning interferometry: measurement and correction of three-dimensional transfer and point-spread characteristics," Appl. Opt. 53(8), 1554-1563 (2014).

[17] Su, R., Thomas, M., Leach, R. K. and Coupland, J. M., "Effects of defocus on the transfer function of coherence scanning interferometry," Opt. Lett. 43(1), 82-85 (2018).

[18] Coupland, J. M. and Lobera, J., "Holography, tomography and 3D microscopy as linear filtering operations," Meas. Sci. Technol. 19(7), 074012 (2008).

[19] Beckmann, P. and Spizzichino, A., [The Scattering of Electromagnetic Waves from Rough Surfaces], Artech House, Norwood, MA, USA (1987).

[20] Sheppard, C. J. R., "Imaging of random surfaces and inverse scattering in the Kirchoff approximation," Waves in Random Media 8(1), 53-66 (1998).

[21] Su, R., Thomas, M., Liu, M., Benketaf, S., Drs, J., Bellouard, Y., Pruss, C., Coupland, J. M., and Leach R. K., "Improved surface measuring interference microscope through error compensation in three-dimensional fringe data," Under preparation.

[22] McCutchen, C. W., "Generalized aperture and the three-dimensional diffraction image," J. Opt. Soc. Am. 54(2), 240-244 (1964).

[23] Goodman, J. W., [Introduction to Fourier Optics, 4th ed.], W. H. Freeman, New York (2017).

[24] de Groot, P. and Deck, L., "Surface profiling by analysis of white-light interferograms in the spatial frequency domain," J. Mod. Opt. 42(2), 389-401 (1995).

[25] Bellouard, Y., Said, A., Dugan, M. and Bado, P., "Fabrication of high-aspect ratio, micro-fluidic channels and tunnels using femtosecond laser pulses and chemical etching," Opt. Express 12(10), 2120-2129 (2004).

[26] Drs, J., Kishi, T. and Bellouard, Y., "Laser-assisted morphing of complex three dimensional objects," Opt. Express 23(13), 17355-17366 (2015).

[27] Lehmann, P., Tereschenko, S. and Xie, X., "Fundamental aspects of resolution and precision in vertical scanning white-light interferometry," Surf. Topogr. - Metrol. Prop. 4, 024004 (2016). 